\title{
FGF1 goes long to tackle diabetes
}

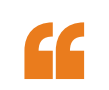

\section{parenteral \\ administration \\ of rFGF 1}

could be a

novel diabetic

therapy

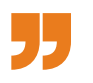

Research published in Nature has found that subcutaneous delivery of fibroblast growth factor 1 (FGF1) confers novel insulin-sensitizing properties to this molecule in diabetic mice while avoiding many of the side effects associated with current antidiabetic drugs.

Thiazolidinediones are highly effective agents for improving insulin sensitivity in patients with type 2 diabetes, and act by switching on the nuclear receptor peroxisome proliferator-activated receptor- $\gamma$ $(\operatorname{PPAR} \gamma)$, which regulates gene networks involved in lipid and glucose handling. The use of these drugs is limited, however, by serious adverse effects including bone loss and liver steatosis ('fatty liver').

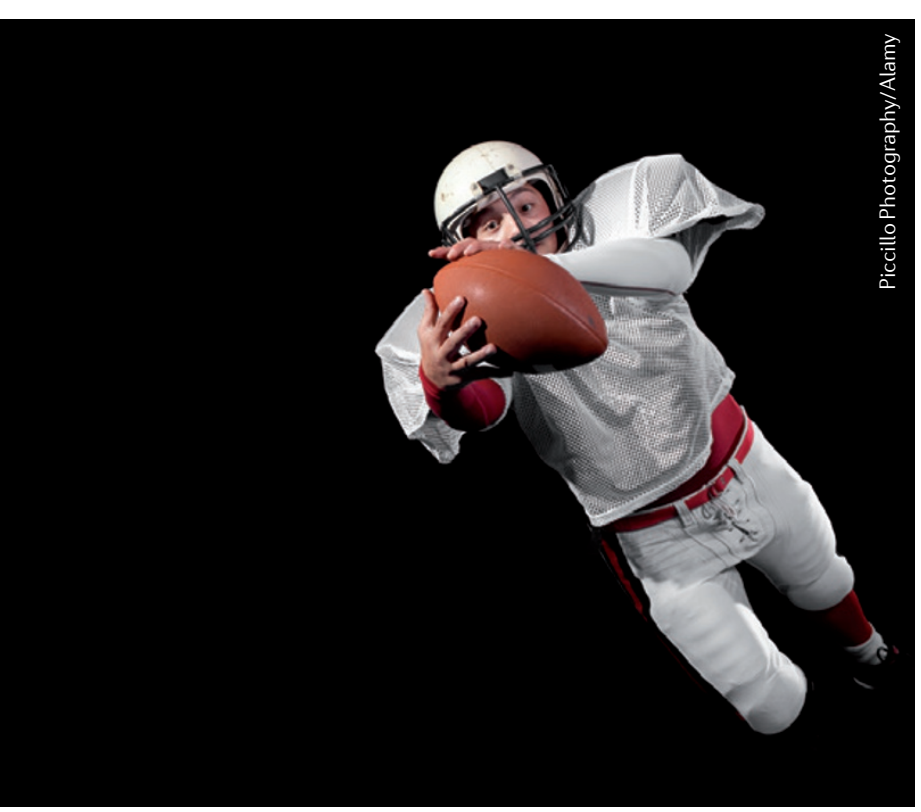

FGF1 is a mitogenic signalling molecule that is regulated by PPAR $\gamma$. Normally, it acts locally in an autocrine and paracrine fashion as it rapidly binds to heparan sulphate proteoglycans in the extracellular matrix. Several lines of evidence have suggested a role for the FGF family of proteins in glucose homeostasis, including the observation of insulin resistance in FGF1-knockout mice stressed by a high-fat diet. In the current study, Suh et al. set out to determine whether enabling FGF1 to signal in an endocrine fashion by administering it parenterally - could be beneficial for treating diabetes.

The researchers subcutaneously injected recombinant murine FGF1 (rFGF1) into genetic and diet-induced obese mouse models of diabetes, and observed rapid glucose lowering that was sustained for 48 hours. Even at the highest dose (2 mg per $\mathrm{kg}$ ), hypoglycaemia was not induced, supporting the potential safety of this approach. Moreover, compared with vehicletreated diabetic mice, chronic (35-day) treatment with rFGF1 significantly improved performance in a glucose tolerance test and an insulin tolerance test. Such changes were associated with decreased circulating levels of inflammatory cytokines, as well as increased hepatic and peripheral insulin sensitivity. Taken together, the results suggest that parenteral administration of rFGF1 could be a novel diabetic therapy.
Importantly, chronic treatment with rFGF1 did not impair bone density or structure - problems that have been associated with the endocrine FGF21 - and did not cause weight gain or liver steatosis. To address the concern that the mitogenicity of FGF1 could give rise to adverse effects, Suh et al. developed an FGF1 ligand that lacked the first 24 residues from the amino terminus. In vitro, this compound showed significantly decreased mitogenic activity compared with full-length FGF1, but retained the glucoselowering effect in mouse models. This work suggests that the growthinducing effect of FGF1 can be dissociated from its antidiabetic action.

FGF1 can signal through all alternatively spliced forms of FGF receptors (FGFRs), but which subtype is responsible for the glucoselowering effects of rFGF1? Previous work highlighted FGFR1 as having a role in regulating insulin sensitivity. Indeed, in the current study, transgenic mice lacking Fgfr1 predominantly in adipose tissue did not show the glucose-lowering response to rFGF1, thus confirming the identity of the key underlying receptor.

Together, the findings illustrate a novel side to FGF1 signalling that could provide substantial and longlasting sensitization to insulin for the treatment of diabetes.

Katie Kingwell

ORIGINAL RESEARCH PAPER Suh, J. M. et al. Endocrinization of FGF1 produces a neomorphic and potent insulin sensitizer. Nature http://dx.doi. org/10.1038/nature13540 (2014) 